\title{
Physiological adaptations of Saccharomyces cerevisiae evolved for improved butanol tolerance
}

\author{
Payam Ghiaci, Joakim Norbeck and Christer Larsson ${ }^{*}$
}

\begin{abstract}
Background: Butanol is a chemical with potential uses as biofuel and solvent, which can be produced by microbial fermentation. However, the end product toxicity is one of the main obstacles for developing the production process irrespective of the choice of production organism. The long-term goal of the present project is to produce 2-butanol in Saccharomyces cerevisiae. Therefore, unraveling the toxicity mechanisms of solvents such as butanol and understanding the mechanisms by which tolerant strains of S. cerevisiae adapt to them would be an important contribution to the development of a bio-based butanol production process.

Results: A butanol tolerant S. cerevisiae was achieved through a series of sequential batch cultures with gradual increase of 2-butanol concentration. The final mutant (JBA-mut) tolerates all different alcohols tested at higher concentrations compared to the wild type (JBA-wt). Proteomics analysis of the two strains grown under mild butanol-stress revealed 46 proteins changing their expression by more than 1.5-fold in JBA-mut, 34 of which were upregulated. Strikingly, 21 out of the 34 upregulated proteins were predicted constituents of mitochondria. Among the non-mitochondrial up-regulated proteins, the minor isoform of Glycerol-3-phosphatase (Gpp2) was the most notable, since it was the only tested protein whose overexpression was found to confer butanol tolerance.

Conclusion: The study demonstrates several differences between the butanol tolerant mutant and the wild type. Upregulation of proteins involved in the mitochondrial ATP synthesizing machinery constituents and glycerol biosynthesis seem to be beneficial for a successful adaptation of yeast cells to butanol stress.
\end{abstract}

Keywords: Saccharomyces cerevisiae, Butanol, Tolerance, Proteomics

\section{Background}

There is a necessity of finding substitutes for finite fossil resources in production of fuels as well as various chemicals that nowadays are obtained as petroleum derivatives. Butanol is a class of chemical products which has several applications such as fuel, industrial solvent etc. [1] and it has several advantages in comparison with ethanol as a biofuel, such as higher energy density, lower water content and vapor pressure [1-3]. It has mostly been produced as a petrochemical but there are also biological alternatives available $[4,5]$. Both native and engineered microorganisms have been used as butanol producers [1,6-11]. Some species of Clostridium genus (e.g. C. acetobutylicum and C. beijerinckii) are known to produce 1-butanol naturally, mixed with acetone and ethanol [6]. However, the limited availability of genetic

\footnotetext{
* Correspondence: christer.larsson@chalmers.se

Department of Chemical and Biological Engineering, System and Synthetic Biology, Chalmers University of Technology, Gothenburg, Sweden
}

tools makes Clostridia less competitive compared to species like Escherichia coli and S. cerevisiae where genetic manipulation techniques are well developed with regards to successful heterologous protein expression. S. cerevisiae in particular is a well-studied organism with a long history of industrial use; e.g. in brewing and ethanol production. Several groups have engineered S. cerevisiae to produce 1-butanol and isobutanol either through redirecting amino acid biosynthetic pathways or by introducing the 1-butanol pathway of C. acetobutylicum [12,13]. The same strategies have been applied to produce 1-butanol and isobutanol in E. coli [8-10]. However, for all production organisms, natural or engineered, end product toxicity is one of the main limiting factors in developing an effective butanol production process [14], i.e. the tolerance level towards butanol is rather similar for Clostridia and S. cerevisiae [14-16]. Different isomers of butanol exhibit different extents of toxicity. 1-butanol being the most toxic with concentrations above $1.5-2 \%$ 
(v/v) being inhibitory for most cells including the native 1-butanol producers $[14,16,17]$ while iso-butanol and 2butanol can be tolerated at higher levels $[12,18]$. One of the reasons for this is probably that 1 -butanol is the most hydrophobic molecule with the strongest ability to permeate and/or interact with the cellular membrane [19-26]. Different alcohol toxicity mechanisms have been described such as accumulation of toxic metabolites [27], changes in membrane fluidity and transport disturbance $[19,20,22-26,28-30]$ as well as disorders in translation initiation [31].

In this study a strain of $S$. cerevisiae was evolved to become more tolerant towards 2 -butanol by making sequential batch cultures with increasing 2-butanol concentrations. However, the resulting strain was also more tolerant to all alcohols tested. In order to understand the mechanisms that are important for the observed butanol tolerance we compared the protein expression profile, lipid content and growth behavior of the evolved tolerant mutant (JBA-mut) and wild-type $(J B A-w t)$. Results from these studies point to the importance of mitochondria and glycerol synthesis as tolerance determinants.

\section{Results and discussions}

\section{Strain evolution}

A butanol tolerant yeast (S. cerevisiae) was selected by evolution under butanol stress. The starting yeast strain $(J B A-w t)$ which is inherently quite ethanol tolerant [32] was cultivated in sequential batches while exposed to stepwise increases in concentrations of 2-butanol $(1.9 \%$, $2.5 \%$ and $3 \% \mathrm{v} / \mathrm{v})$. Transfer of the strain to a fresh media with higher 2-butanol concentration was done provided that growth rate in the previous concentration had been constant for several transfers. After a total of 30 serial transfers (within 24 days and equivalent to roughly 100 generations) the cells could grow in $3 \% \mathrm{v} / \mathrm{v} 2$-butanol while the wild type strain $(J B A-w t)$ could not sustain growth at this concentration (Figure 1). Our selection pressure was exposure to 2-butanol, but we were also interested in investigating whether this strain would display an increased tolerance to the other isomers of butanol as well as ethanol and propanol, or whether this ability is exclusive for 2-butanol. The results showed that the evolved strain was more resistant to both 1-butanol and iso-butanol (Figure 1). In addition, the ethanol tolerance was improved although not to the same extent as for butanol (Figure 1). The tolerance to 1-propanol was also increased (data not shown). Hence, we conclude that the JBA mut strain displays an increased tolerance especially to inhibitory concentrations of higher alcohols and hence might serve as a suitable model to elucidate the cellular adaptation mechanisms leading to alcohol tolerance.

\section{Protein expression profile}

With the evolved tolerant strain (JBA-mut) in hand, it was of obvious interest to investigate into the molecular mechanisms underlying the tolerance phenotype. Different approaches might be chosen to assess physiological adaptations of an evolved strain. In this study we chose to look at the differences on the level of the proteome, lipid composition and physiological properties. Applying a SDS-associated protein extraction coupled to protein trypsin digestion and mass spectrometry (MS) analyses allowed us to also study poorly soluble proteins (e.g. membrane bound proteins). This was important since butanol stress is likely to act via changes in plasma membrane properties, possibly involving changes in the expression of membrane associated proteins, which are known to be difficult to quantify.

Many stresses, including exposure to butanol, lead to a reduced growth rate, which is in turn a major source of gene expression changes [33]. In order to determine a threshold value at which the mutant $(J B A-m u t)$ and the wild type $(J B A-w t)$ strain exhibited a similar growth rate we performed a screen of the growth rate of our mutant strain and the corresponding wild type strain in a range of 2-butanol concentrations. The highest concentration of 2-butanol at which the growth rates of the two strains was identical was determined to be approximately $1.2 \%$ $(\mathrm{v} / \mathrm{v})$, which was therefore chosen as the condition for cultivation and harvest of cells for protein extraction and MS analysis. At this concentration of 2-butanol, an approximately $10 \%$ reduction in growth rate was seen for both strains, indicative of cellular stress by 2 butanol. Cells were grown in biological triplicates in $1.2 \%(\mathrm{v} / \mathrm{v}) 2$-butanol and harvested at $\mathrm{OD}_{600} \approx 1$ (corresponding to roughly $1 \times 10^{7}$ cells $/ \mathrm{ml}$ ), a point at which exponential growth would still continue for about two generations and nutrient limitation would not be a major problem. Following the harvest, proteins were extracted, digested with trypsin and labeled with TMTreagents (as described in the materials and method section). In the subsequent LC-coupled mass spectrometric analysis, we were able to quantify approximately 1100 proteins from the $J B A-w t$ and $J B A-m u t$ strains. These 1100 proteins contained representatives from essentially all cellular locations (notably also many plasma membrane proteins), which we take as verification for the utility of our choice of extraction and trypsination method. Most components of the central metabolic pathways were quantified, as well as essentially all components of the ribosomes, together with many representatives of structural and regulatory factors. It is likely that many more proteins of lower abundance could have been quantified in a second round of targeted mass spectrometric analysis, by actively avoiding the peptides analyzed in the first round. However, given the rather clear conclusion from 
the primary analysis of protein expression (see below) we decided to not go on with such an analysis.

The expression of ribosomal proteins are usually good indicators of changes in growth rate [33] and we therefore first looked at the expression of these proteins. In good agreement with our choice of culture conditions giving a similar growth rate for both strains, it was found that the expression of ribosomal proteins did not change significantly (an average mutant/wt expression change of $0.93+/-0.06$ for $40 \mathrm{~S}$-subunits and $0.92+/-$ 0.12 for the $60 \mathrm{~S}$-subunits). This result indicates that any changes in protein expression would most likely not be caused by differences in growth rate.
We next decided to narrow down the data on quantification of $\sim 1100$ proteins by a Student's t-test (using a $\geq 95 \%$ significance threshold). 34 proteins were found with at least 1.5 fold up-regulations (Table 1) while 12 proteins were down-regulated 1.5 fold or more by the same criteria (Table 2) when comparing the JBA-mut to the JBA-wt strain. Most strikingly, 21 out of 34 upregulated proteins were mitochondrially associated, among which 12 were components of respiratory chain including ATP-synthase and cytochrome $\mathrm{c} 1$ which is clearly a highly significant enrichment. The fact that about two-thirds of the upregulated proteins were mitochondrial is a strong indication of higher mitochondrial

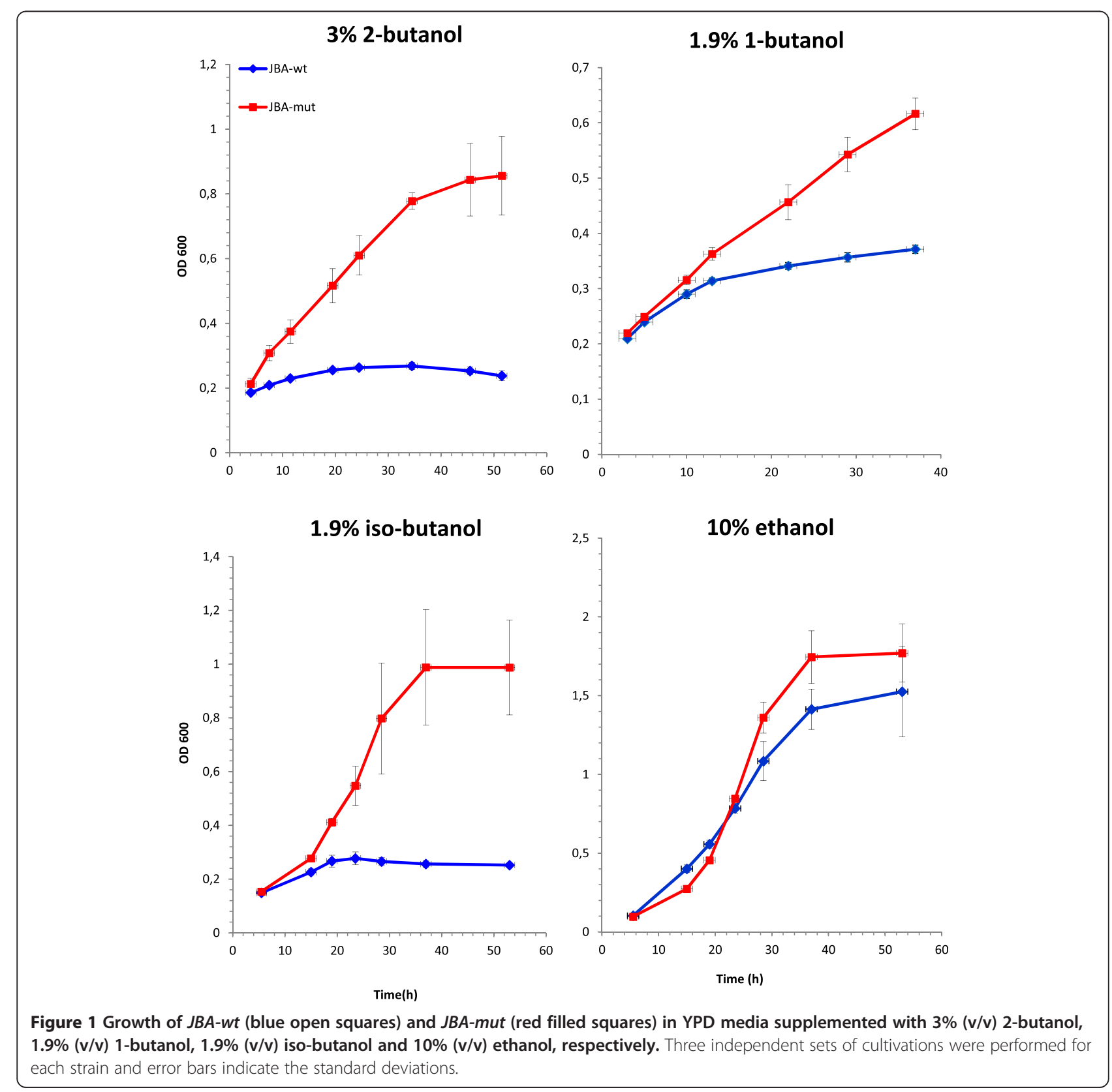


Table 1 Proteins showing at least 1.5 fold overexpression with $95 \%$ significance in JBA-mut compared to JBA-wt

\begin{tabular}{|c|c|c|c|}
\hline Gene & Corresponding protein & Fold change & Mitochondrial location \\
\hline PIM1 & Lon protease homolog & 2.86 & $\mathrm{x}$ \\
\hline MAM33 & Mitochondrial acidic protein MAM33 & 2.72 & $\mathrm{x}$ \\
\hline CYT1 & Cytochrome $\mathrm{cl}$, heme protein & 2.09 & $\mathrm{x}$ \\
\hline GLO1 & Lactoylglutathione lyase & 2.01 & \\
\hline HSP42 & Heat shock protein 42 & $1.97 *$ & \\
\hline AIM2 & Protein AIM2 & 1.94 & $\mathrm{x}$ \\
\hline FUN30 & Uncharacterized ATP-dependent helicase FUN30 & $1.88 *$ & $\mathrm{x}$ \\
\hline HOR2/GPP2 & (DL)-glycerol-3-phosphatase 2 & $1.81 *$ & \\
\hline MCR1 & NADH-cytochrome b5 reductase 2 & $1.80 *$ & $\mathrm{x}$ \\
\hline GLK1 & Glucokinase-1 & $1.74 *$ & \\
\hline MRPL38 & $54 S$ ribosomal protein $L 38$ & $1.70 *$ & $\mathrm{x}$ \\
\hline QCR6 & Cytochrome b-c1 complex subunit 6 & 1.67 & $\mathrm{x}$ \\
\hline EDE1 & EH domain-containing and endocytosis protein 1 & 1.65 & \\
\hline MSS116 & ATP-dependent RNA helicase MSS116 & $1.65 *$ & $\mathrm{x}$ \\
\hline YPL088W & Putative aryl-alcohol dehydrogenase YPL088W & $1.64 *$ & \\
\hline ATP4 & ATP synthase subunit 4 & 1.62 & $\mathrm{x}$ \\
\hline ATP17 & ATP synthase subunit $f$ & $1.62 *$ & $\mathrm{x}$ \\
\hline PEP4 & Saccharopepsin & 1.62 & \\
\hline LSP1 & Sphingolipid long chain base-responsive protein LSP1 & 1.62 & \\
\hline QCR2 & Cytochrome b-c1 complex subunit 2 & 1.61 & $\mathrm{x}$ \\
\hline $\operatorname{cox} 4$ & Cytochrome c oxidase subunit 4 & 1.59 & $\mathrm{x}$ \\
\hline ZWF1 & Glucose-6-phosphate 1-dehydrogenase & 1.59 & \\
\hline ECM33 & Cell wall protein ECM33 & 1.58 & \\
\hline GVP36 & Protein GVP36 & 1.57 & \\
\hline CCP1 & Cytochrome c peroxidase & 1.57 & $\mathrm{x}$ \\
\hline CAR2 & Ornithine aminotransferase & $1.57 *$ & \\
\hline AAC2 & ADP, ATP carrier protein 2 & 1.56 & $x$ \\
\hline $\mathrm{CYC1}$ & Cytochrome c iso-1 & $1.56 *$ & $x$ \\
\hline ATP1 & ATP synthase subunit alpha & $1.55 *$ & $x$ \\
\hline ATP2 & ATP synthase subunit beta & $1.54 *$ & $x$ \\
\hline CPR3 & Peptidyl-prolyl cis-trans isomerase C & 1.54 & $\mathrm{x}$ \\
\hline KGD1 & 2-oxoglutarate dehydrogenase & 1.54 & $x$ \\
\hline QCR7 & Cytochrome b-c1 complex subunit 7 & 1.53 & $x$ \\
\hline MRP8 & Uncharacterized protein MRP8 & $1.51 *$ & \\
\hline
\end{tabular}

14 proteins remain upregulated also with $99 \%$ significant t-test (marked with asterisks).

activity in JBA-mut. This might be explained as an effort to compensate high ATP consumption rates due to malfunction of the plasma membrane barrier or poor performance of ATP synthesis. Among the nonmitochondrial proteins most upregulated in the JBA-mut strain we found Lactoylglutathione lyase (Glo1), the small heat shock protein (Hsp42), the osmotically regulated glycerol 3-phosphatase (Hor2/Gpp2) and Glucokinase I (Glk1). Hor2/Gpp2 is one of two highly similar isogenes coding for the last step in glycerol biosynthesis
[34] and it is also part of the general stress response [33]. The upregulation of Hor2/Gpp2 is interesting in view of the fact that upregulation of the highly similar isogene $R H R 2 / G P P 1$ was found to be a strain specific determinant (SSG) of ethanol tolerance [35], suggested to mediate an increased capacity for NADH oxidation. A need for NADH oxidation would also be compatible with the observed increase in expression of proteins in the mitochondrial respiratory chain in the JBA-mut strain. However, similar to the study by Stanley et al. 
Table 2 Proteins showing at least 1.5 fold down-regulation with $95 \%$ significance in JBA-mut compared to JBA-wt

\begin{tabular}{lcc}
\hline Gene & Corresponding protein & Fold change \\
\hline TY2A-GR1 & Transposon Ty2-GR1 Gag polyprotein & 0.24 \\
RPL7B & 60S ribosomal protein L7-B & $0.39 *$ \\
MET6 & 5-methyltetrahydropteroyltriglutamate-homocysteine methyltransferase & $0.50 *$ \\
HIS1 & ATP phosphoribosyltransferase & 0.51 \\
HR11 & Protein HRI1 & 0.56 \\
TDH2 & Glyceraldehyde-3-phosphate dehydrogenase 2 \\
SHM2 & Serine hydroxymethyltransferase, cytosolic & $0.57 *$ \\
RTN1 & Reticulon-like protein 1 & 0.59 \\
PDC5 & Pyruvate decarboxylase isozyme 2 & 0.60 \\
STE20 & Serine/threonine-protein kinase STE20 & 0.62 \\
FSH1 & Family of serine hydrolases 1 & 0.65 \\
NOP16 & Nucleolar protein 16 & 0.66 \\
\hline
\end{tabular}

4 proteins remain downregulated also with $99 \%$ significant t-test (marked with asterisks).

[35], we do not observe any significant change in any of the other glycerol metabolic enzymes, even though a slightly increased yield of glycerol was found (see below). Glo1 is involved in the detoxification of methylglyoxal the increased production of which is commonly seen under many forms of stress, notably during conditions of increased glycerol production [36]. The expression of glucokinase I (Glk1) is frequently found under stress conditions [33], but since the expression of the more abundant Hxk2 protein did not change, the significance of this is unclear.

In $E$. coli the chaperone GroESL has been implicated as a factor in butanol tolerance [37]. We do not see a strong upregulation of the yeast homolog of GroEL (Hsp60), but the most upregulated protein in our study is the Lon protease homolog Pim1, which is involved in degradation of misfolded protein in the mitochondrial matrix [38]. The cytoplasmic heat shock protein Hsp42 was also found as a highly significant, 2-fold induced protein in the JBA-mut strain. Hsp42 has a role in sorting of peripheral protein aggregates in S. cerevisiae under non-heat shock conditions causing protein folding defects, thereby aiding in maintaining protein homeostasis [39]. We have not looked into the formation of protein aggregates in our strain, but from analogy to the situation in E. coli we find it likely that butanol stress would lead to problems of protein folding and hence to aggregate formation, thereby providing a plausible explanation for the upregulation of Hsp42 and Pim1.

There were also 12 proteins showing a significant ( $95 \%$ confidence level by Student's t-test) and $\geq 1.5$ fold reduced abundance in the JBA-mut strain (Table 2). The protein showing the largest change was a transposon Ty2 Gag polyprotein, which could indicate that a transposon of this type has moved to a new location. However, although there are usually many transposons in the yeast genome [40], this was the only transposon associated protein detected in our samples, and the biological relevance of this finding is therefore hard to judge. We are planning to sequence the genomes of the JBA-mut and JBA-wt strains which might shed some light on this issue. The second most repressed protein was Rpl7B, a component of the large subunit of the ribosome. This was the only ribosomal protein showing a significant change in expression of $>1.5$ fold in any direction. Rpl7B is highly similar to Rpl7A which shows no change in expression. The significance of this differential expression is unclear, even though it has previously been suggested that the two isoforms may have somewhat different roles in the cell [41]. The repressed proteins Met6, His1 and Shm2 are all involved in amino acid biosynthesis indicating that there could be specific changes of fluxes over these reactions. However, the vast majority of amino acid biosynthetic enzymes do not display a significantly changed abundance in the JBA-mut. There are also two minor isoforms of proteins in the glycolysis and ethanolsynthetic pathway showing a lowered abundance in the $J B A-m u t$ strain: glyceraldehyde-3-phosphate dehydrogenase (Tdh2) and pyruvate decarboxylase (Pdc5). However, the corresponding dominant isoforms of these enzymes (Tdh3 and Pdc1) show no significant change in expression. Overall, the mechanistic significance of the proteins showing reduced abundance is thus unclear. Changing the t-test significance to $\geq 99 \%$ results in 14 proteins upregulated ( 8 of which are mitochondrial) and 4 proteins down-regulated at least 1.5 fold (Table 1 and Table 2).

In summary, the main hypothesis emerging from the proteomics analysis is that a somewhat higher mitochondrial activity, perhaps together with a higher glycerol biosynthesis and a selection of stress response proteins (e.g. Hsp42 and Glo1) is the main factor underlying the 
butanol tolerant phenotype. This is in good agreement with previously published data on ethanol tolerance determinants which have also underscored the role of mitochondria $[35,42,43]$. Furthermore, protein degradation and retrograde signalling have recently been suggested as key factors in mediating butanol tolerance in yeast [44]. We find no evidence for increased proteolysis in our data, but it is possible that the increase we observe in mitochondrial proteins could be mechanistically linked to retrograde signalling as proposed by Gonzalez-Ramos et al. [44].

\section{Improvement of butanol tolerance by over expression of selected targets proposed from proteomics data}

To test the hypothesis that the upregulated proteins were at least in part responsible for the increased butanol tolerance of the mutant strain $(J B A-m u t)$, we decided to investigate into whether individual over-expression of the three most strongly regulated, non-mitochondrial, proteins (Gpp2, Glo1 and Hsp42; see Table 1) would lead to a tolerant phenotype. Overexpression was achieved by genomic integration of a construct in which the gene expression was placed under the control of a strong TDH3 promotor (see Methods-section). The results showed that only the overexpression of GPP2 mediated an increase in butanol tolerance at a 2-butanol concentration of $3 \%(\mathrm{v} / \mathrm{v})$ although, as expected, not to the same level as the mutant (Figure 2). Overexpression of GLO1 and HSP42 did not improve the butanol tolerance of the wild-type strain (data not shown). We also tried overexpression of HAP4 which has been shown to induce an increase in respiration [45]. For unknown reasons we were unable to overexpress HAP4 in the JBA-wt strain, but the same construct when introduced into a wild type CEN_PK strain did not provide a butanol tolerant phenotype. This could indicate that either (i) the increase in mitochondrial activity proposed by the proteomics data is dependent on synergy with other changes in gene expression, presumably not regulated via Hap4 and furthermore not present in the haploid CEN_PK strain, or (ii) that the change in mitochondrial activity could be a consequence of the changes in gene/protein expression mediating the butanol tolerance. The synergy hypothesis would explain why most of the tested overexpressions did not lead to a tolerance phenotype, but it would also suggest studying overexpression of combinations of two or more proteins simultaneously which would rapidly turn into a daunting task given the number of candidate genes. However, since increased Gpp2 expression seems to be a contributing factor to butanol tolerance, it will be interesting in future studies to clarify the role of glycerol metabolism and its connection to mitochondrial processes (and other proteins found in this study) in mediating butanol tolerance.

Thus, since increased Gpp2 expression seems to be a contributing factor to butanol tolerance, it will be interesting in future studies to clarify the role of glycerol metabolism and its connection to mitochondrial processes in mediating butanol tolerance.

\section{Lipid analysis}

Several studies have shown the connection of alcoholstress and membrane lipid composition of cells [22,25,46-48]. We therefore decided to investigate whether the mutant phenotype was at least in part also dependent on changes in the abundance of one or

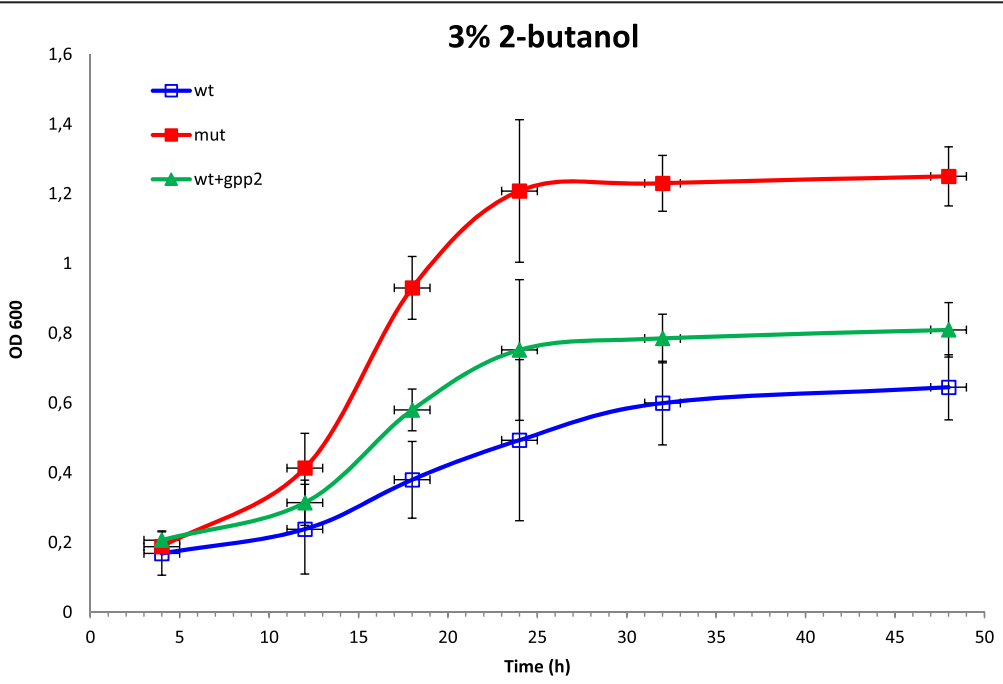

Figure 2 Growth of JBA-wt (blue open squares), JBA-wt + TDH3promotor-GPP2 (green closed triangles) and JBA-mut (red closed squares) in YPD media supplemented with $3 \%(\mathbf{v} / \mathbf{v})$ 2-butanol, respectively. Three independent sets of cultivations were performed for each strain and error bars indicate the standard deviations. 
several different classes of lipids. Following the procedure described by Khoomrung et al. [49] we could extract the lipids and fatty acids by using a single extraction. Similar to the proteomics samples the cells were grown in YPD with $1.2 \% \mathrm{v} / \mathrm{v}$ 2-butanol and harvested at $\mathrm{OD}_{600} \approx 1$. There was a general trend showing a somewhat increased content of lipids in the JBA mut strain (Figure 3), however, these changes were not found significant by a Student t-test. The largest increase was obtained for phosphatidylethanolamine (PE) and phosphatidylserine (PS) where an increase of about $60 \%$ was observed for the mutant compared to the wild-type.

However, we have not looked at the composition of the constituent fatty acids for each class of lipid. Thus, although the abundance of the various classes of lipids does not change significantly, the properties of the fatty acid composition in some or all classes might do so. These analyses would be interesting to perform as a follow up study. However, at present we find little evidence for involvement of lipids in mediating the tolerant phenotype of the mutant, both from the lack of changes in enzymes in lipid biosynthesis and from direct lipid measurements.

\section{Physiological growth characteristics}

Some differences in growth characteristics were discovered when cultivating $J B A-w t$ and $J B A-m u t$ in bioreactors, in the presence of $1.2 \%(\mathrm{v} / \mathrm{v})$ 2-butanol. As described before, the growth rate at this butanol concentration was more or less identical between the two strains (Figure 4). The growth yield and glucose consumption rates are also quite similar though there was a slightly reduced sugar consumption rate and somewhat higher final OD of the mutant. There was also a small difference in fermentation rates as the mutant displayed reduced ethanol production rate compared to the wildtype (Figure 4). This is consistent with the proteomics data which indicated a higher mitochondrial activity of the mutant. Another observation that could be related to changes in protein levels was the increase in glycerol production in the mutant (Figure 4) since glycerol 3phosphatase 2 (Gpp2) was one of the proteins showing the highest and most significant ( $299 \%$ significance) upregulation. In many cases an increase in ethanol production rate is also accompanied by an increase in glycerol formation [50] but in this case the evolved tolerant mutant may have induced an inherent higher glycerol production rate as a general stress response.

It should be also mentioned that the JBA-mut did not display a butanol tolerant phenotype during growth under anaerobic conditions (data not shown). An increased glycerol production rate is well known to occur under anaerobic conditions [51], but the restricted ATP synthesizing capacity under this condition most likely prevents the ability to tolerate high butanol concentrations.

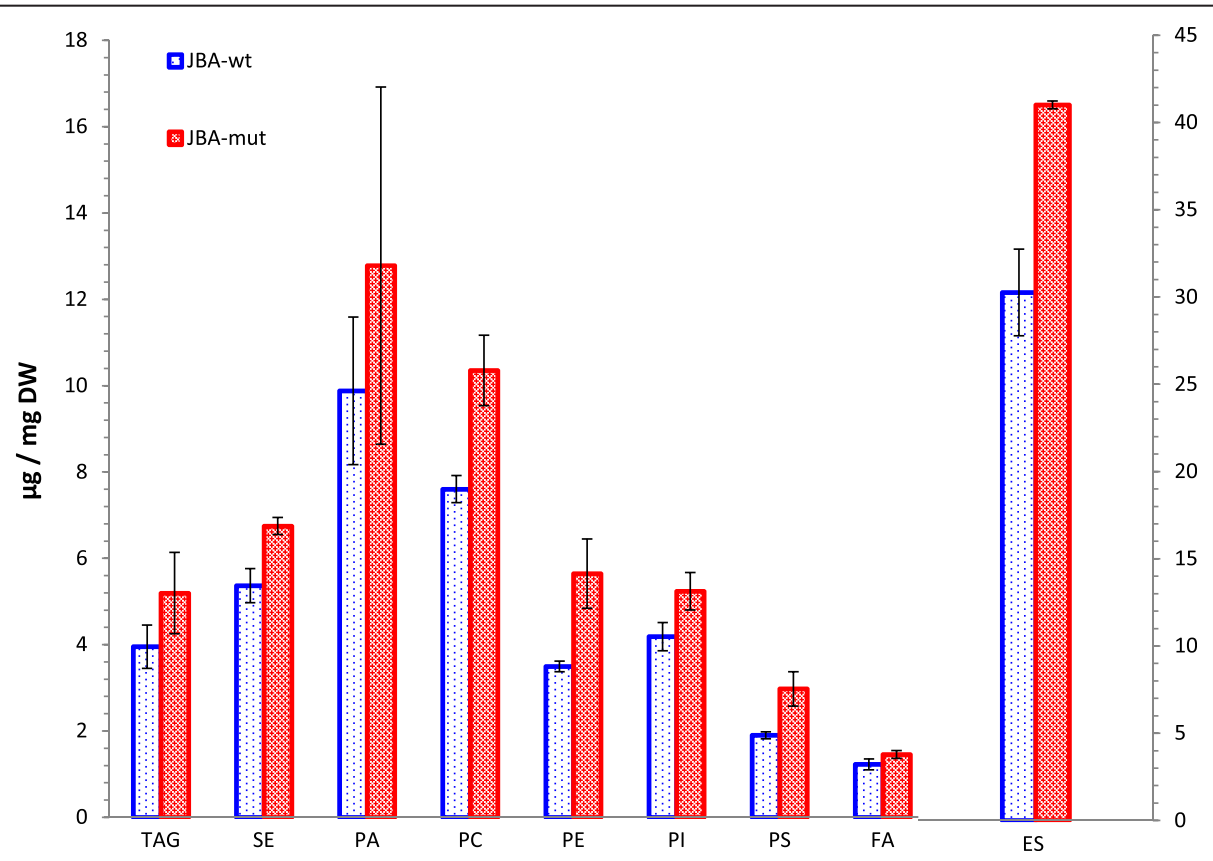

Figure 3 Lipid and fatty acid composition of JBA-wt (blue open bars) and JBA-mut (red filled bars) during growth in YPD with $1.2 \%$ (v/ v) 2-butanol. Two independent sets of cultivations were performed for each strain and error bars indicate the min/max values. (TAG triacylglycerol, SE - steryl ester, PA - Phosphatidic acid, PC - Phosphatidylcholine, PE - phosphatidyletanolamine, PI - phosphatidylinositol, PS phosphatidylserine, FA - free fatty acid, ES - ergosterol). 

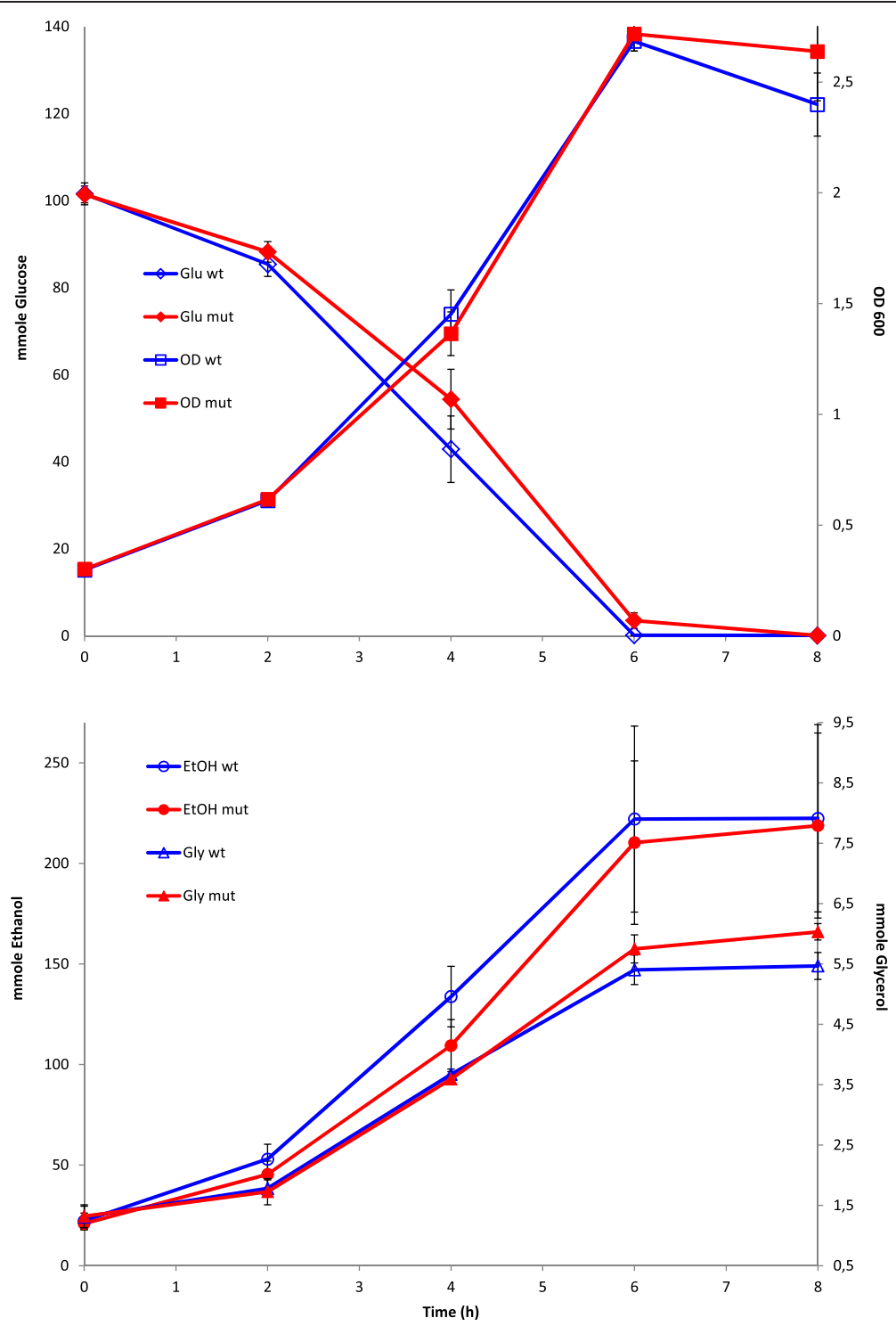

Figure 4 Growth characteristics of $J B A$-wt (blue open symbols) and JBA-mut (red closed symbols). Cells were grown in YPD, in the presence of 1.2\% 2-butanol ( $\mathrm{V} / \mathrm{V}$ ). Measured variables were optical density (squares), glucose (diamonds), ethanol (circles) and glycerol (triangles). Two independent sets of cultivations were performed for each strain and error bars indicate the min/max values.

\section{Conclusion}

The study showed that the wild-type and the mutant differed in a number of aspects. Based on the proteomics data, which are consistent with the physiological characterization, higher mitochondrial activity of the $J B A-m u t$, together with an increased expression of the glycerol 3-phosphatase isoform Gpp2 and increased expression of some of the stress metabolic enzymes (e.g. Glo1 and Hsp42), seem to be the main characteristics providing the JBA-mut with the observed increase in butanol tolerance. The mechanistic explanation for the tolerance phenotype is likely to depend on synergistic effects of several regulatory changes, which we at present do not fully understand, but which are presently being studied in our group.

The fact that overexpression of GPP2 in JBA-wt helped it to adapt to butanol stress further corroborates the role of Gpp2.

The tolerance mechanisms proposed in the present work would lead to a somewhat higher flow of carbon to glycerol and likely also to respiration, thereby to some extent reducing the yield of butanol. However, in the case of most biofuel production processes, the end product toxicity has been identified as a major problem [14]. Therefore, in an industrial process to produce 2-butanol, this could be acceptable, since even a rather modest 
increase in tolerance to butanol would have a significant value, due to the rather high toxicity of 2-butanol.

\section{Methods}

\section{Strain}

An industrial strain of $S$. cerevisiae, obtained from the Baker's Yeast Company in Rotebro, Sweden, was used as the starting strain $(J B A-w t)$.

\section{Cultivation}

The cells were grown in YPD medium $(20 \mathrm{~g} / \mathrm{l}$ glucose, $20 \mathrm{~g} / \mathrm{l}$ peptone and $10 \mathrm{~g} / \mathrm{l}$ yeast extract). Through $30 \mathrm{se}-$ quential batch cultures in $50 \mathrm{ml}$ falcon tubes the mutated strain was obtained. Butanol concentration was gradually increased along the transfers and prior to each transfer, cells were recovered in fresh YPD medium for 30 minutes. Each time $0.5 \mathrm{ml}$ of culture (approximately $1.5 \times 10^{7}$ cells) was transferred.

Later on the wild type $(J B A-w t)$ and the mutant $(J B A-$ $m u t$ ) strains were cultivated in bioreactors (Dasgip, Jülich, Germany) (in the presence of $10 \mathrm{~g} / \mathrm{l}$ 2-butanol) for characterization. The medium was stirred at $400 \mathrm{rpm}$ and no air was purged to avoid stripping of butanol.

\section{Strain construction and transformation}

The open reading frames from the genes of interest (i.e. GPP2, GLO1 and HSP42) were integrated into the genome of $J B A-w t$ using an integrative construct (provided as Additional file 1) at a locus $1000 \mathrm{bp}$ downstream of the DAK2 gene stop-codon with the Kanamycin resistance marker (KanMX4) used to isolate correct colonies. Standard lithium acetate protocol was used for transformation.

\section{MS sample preparation}

$80 \mu \mathrm{l}$ of cell pellets were mixed with $70 \mu \mathrm{l} 100 \mathrm{mM}$ Triethylammoniumbicarbonate (TEAB) solution to keep the $\mathrm{pH}$ stable at about $8.50 \mu \mathrm{l}$ glassbeads was added and the sample was mixed vigorously by Fast Prep (MP Biomedicals Solon, OH, USA) to break the cells (20 seconds at speed 6 for 4 times, kept 30 seconds on ice between every run). $30 \mu \mathrm{l} 10 \%$ SDS was added and the samples were heated at $60^{\circ} \mathrm{C}$ for 5 minutes. The samples were centrifuged at high speed for 2 minutes and the supernatant was kept.Total protein concentration was determined using Pierce $660 \mathrm{~nm}$ Protein Assay (Thermo Scientific). Three samples in each group (mutant and wildtype) containing $100 \mu \mathrm{g}$ protein in each sample were incubated with TCEP (tris(2-carboxyethyl)phosphine) and then transferred to $3 \mathrm{~K} \mathrm{mw}$ cutoff filters (PALL) and diluted with $8 \mathrm{M}$ urea in $0.1 \mathrm{M}$ TEAB (triethyl ammonium bicarbonate) for filter aided sample preparation (FASP) [52].
Samples were alkylated with MMTS (methyl methanethiosulfonate) and digested with trypsin in $0.5 \mathrm{M}$ TEAB ratio 1:25 over night in $37 \mathrm{C}$. The peptides were eluted at $12000 \mathrm{rpm}$ for $10 \mathrm{~min}$ and filters were rinsed with 20\% ACN (acetonitrile) at $12000 \mathrm{rpm}$ for 5 min.

\section{Label with TMT reagents}

TMT reagents 126, 128 and 130 (wildtype samples) and 127, 129 and 131 (mutant samples) was dissolved in $\mathrm{ACN}$ and added to the respectively sample according to manufacturer's protocol (Thermo Fisher Scientific). After labelling and quenching of the reagents, the samples were combined and concentrated. TMT-labelled peptides were separated with Strong Cation Exchange Chromatography (SCX). The concentrated peptides were acidified by $10 \%$ formic acid and diluted with SCX solvent A (25 mM ammonium formate, pH 2.8, 20\% ACN and injected onto a PolySULFOETHYL A SCX column $(2.1 \mathrm{~mm}$ i.d. $\times 10 \mathrm{~cm}$ length, $5 \mu \mathrm{m}$ particle size, $300 \AA$ pore size). SCX chromatography and fractionation was carried out on an ÄKTA purifier system (GE healthcare) at $0.25 \mathrm{~mL} / \mathrm{min}$ flow rate using the following gradient: $0 \% \mathrm{~B}$ (500 mM ammonium formate, $\mathrm{pH} 2.8,20 \% \mathrm{ACN}$ ) for $5 \mathrm{~min}$; $0-20 \%$ B for $20 \mathrm{~min}$; 20-40\% B for $10 \mathrm{~min}$ and $40-100 \% \mathrm{~B}$ for $10 \mathrm{~min}$. UV absorbance at 254 and $280 \mathrm{~nm}$ was monitored while fractions were collected at $0.5 \mathrm{~mL}$ intervals and dried down in a SpeedVac. The 18 peptide containing fractions were desalted on PepClean C18 spin columns according to manufacturer's instructions (Thermo Fisher Scientific).

\section{LC-MS/MS analysis on LTQ-OrbitrapXL}

The desalted and dried fractions were reconstituted into $0.1 \%$ formic acid and analyzed on a LTQ-OrbitrapXL (Thermo Fisher Scientific) interfaced with an in-house constructed nano-LC column. Two-micro liter sample injections were made with an HTC-PAL autosampler (CTC Analytics AG) connected to an Agilent 1200 binary pump (Agilent Technologies). The peptides were trapped on a pre-column ( $40 \times 0.075 \mathrm{~mm}$ i.d.) and separated on a reversed phase column, $200 \times 0.075 \mu \mathrm{m}$. Both columns are packed in-house with $3 \mu \mathrm{m}$ Reprosil-Pur C18-AQ particles. The flow through the analytical column was reduced by a split to approximately $200 \mathrm{nl} / \mathrm{min}$ and the gradient was as followed; $0-6 \mathrm{~min} 0.1 \%$ formic acid, 6-106 min 5-37\% ACN, 0.1\% formic acid, up to $80 \%$ ACN during $3 \mathrm{~min}$ and hold at $80 \%$ for $5 \mathrm{~min}$.

LTQ-OrbitrapXL settings were: spray voltage $1.4 \mathrm{kV}, 1$ microscan for MS1 scans at 60000 resolutions $(\mathrm{m} / \mathrm{z}$ 400), full MS mass range $\mathrm{m} / \mathrm{z} 400-2000$. The LTQOrbitrapXL was operated in a data-dependent mode with one MS1 FTMS scan of precursor ions followed by CID (collision induced dissociation) and HCD (high 
energy collision dissociation), MS2 scans of the three most abundant doubly, triply and quadruply protonated ions in each FTMS scan. The settings for the MS2 were as follows: 1 microscans for HCD-MS2 at 7500 resolution (at $\mathrm{m} / \mathrm{z} 400$ ), mass range $\mathrm{m} / \mathrm{z} 100-2000$ with a collision energy of $50 \%, 1$ microscans for CID-MS2 with a collision energy of $30 \%$. Dynamic exclusion of a precursor selected for MS2 was used for 30s after one repeat, enabling most of the co-eluting precursors to be selected for MS2.

\section{Database search and TMT quantification}

MS raw data files from all SCX fractions for the TMT set were merged for relative quantification and identification using Proteome Discoverer version 1.3 (Thermo Fisher Scientific). Database search was performed by Mascot search engine using the following critera: Saccharomyces cerevisiae in Swissprot protein database (version may 2012), MS peptide tolerance as $10 \mathrm{ppm}, \mathrm{MS} / \mathrm{MS}$ tolerance as $0.5 \mathrm{Da}$, trypsin digestion allowing 1 missed cleavages with variable modifications; methionine oxidation, cysteine methylthiol, and fixed modifications; N-terminal TMTlabel, lysine TMT-label. The detected protein threshold in the software was set to $99 \%$ confidence and identified proteins were grouped by sharing the same sequences to minimize redundancy.

For TMT quantification, the ratios of TMT reporter ion intensities in MS/MS spectra (m/z 126.12, 127.13, 128.13, $129.14,130.14,131.14$ ) from raw data sets were used to calculate fold changes between samples. The average of all three reporters for the wild-type samples was used as the denominator. Only peptides unique for a given protein were considered for relative quantitation, excluding those common to other isoforms or proteins of the same family. The resulting ratios were then exported into Excel (Microsoft) for further data interpretation.

\section{Extraction and analysis of lipids and fatty acids}

Lipids (including steryl ester (SE), ergosterol (ES), triacylglycerol (TAG), phosphatidic acid (PA), phosphatidylethanolamine (PE), phosphatidylcholine (PC), phosphatidylserine (PS) and phosphatidylinositol (PI)) and fatty acids were extracted based on a microwaveassisted method [49]. About $10 \mathrm{mg}$ of freeze-dried cells together with internal standard were mixed with chloroform-methanol. $\mathrm{N}_{2}$ gas was purged and samples were heated up in microwave reaction vessel. Samples were mixed with $0.73 \% \mathrm{w} / \mathrm{v} \mathrm{NaCl}$ solution and centrifuged afterwards. Samples were concentrated under vacuum drying and then resuspended in chloroform-methanol. Detailed procedure can be found in [49].

Lipid analysis was done according to [49] by HPLC (Dionex; ultimate 3000 HPLC system, Germany) and a
CAD detector (Corona; ESA, Chelmsford, MA, USA) streamed with $\mathrm{N}_{2}$ at 35 psi. $2 \mu \mathrm{l}$ of sample was passed through a Luna $5 \mathrm{~mm}$ HILIC $100 \AA(250 \times 4.6 \mathrm{~mm}) \mathrm{LC}$ Column (Phenomenex). The flow rate of $0.8 \mathrm{~mL} / \mathrm{min}$ was applied and temperature was kept at $35^{\circ} \mathrm{C}$. Three solution of (1) hexane-acetic acid (99:1, v/v); (2) acetone-isopropanol-acetic acid (29:70:1, v/v) and (3) water-acetone-isopropanol-acetic acid (9:20:70:1, v/v) were used as mobile phase and $\mathrm{pH}$ was adjusted to 5 by addition of triethylamine $(0.08 \%, \mathrm{v} / \mathrm{v})$.

The sample was run for 45.9 minutes and the starting elution solution was $100 \%$ solvent (1) at $t=0 \mathrm{~min}$. Solvent (2) and solvent (3) were added gradually and fraction of solvent (1) was altered accordingly. The gradient of solvent (2) was as follow: $1 \%(t=5 \mathrm{~min}), 2 \%(t=$ $6 \mathrm{~min}), 3 \%(\mathrm{t}=14 \mathrm{~min}), 5 \%(\mathrm{t}=19-36 \mathrm{~min}), 20 \%(\mathrm{t}=$ $38 \mathrm{~min}), 2 \%(t=40 \mathrm{~min})$ and $0 \%(t=42 \mathrm{~min})$. The gradient of solvent (3) reached the following: $0.5 \%(t=14 \mathrm{~min}), 35 \%$ $(\mathrm{t}=19 \mathrm{~min}), 44 \%(\mathrm{t}=36 \mathrm{~min})$ and $0 \%(\mathrm{t}=38 \mathrm{~min})$. Lipids were quantified using external calibration curves. Lipid standards with known concentrations (ranging from 10 to $1000 \mu \mathrm{g} / \mathrm{mL}$ ) were used.

\section{Analysis of extracellular metabolites}

Metabolites such as glucose, ethanol, glycerol and acetate were analyzed by HPLC (Ultimate 3000, Dionex, Sunnyvale, US) through an Aminex ${ }^{\circ}$ HPX-87H column $(300 \times 7.8 \mathrm{~mm})$ (Bio-Rad, Hercules, CA, USA). $5 \mathrm{mM}$ $\mathrm{H}_{2} \mathrm{SO}_{4}$ was used as eluent at $0.6 \mathrm{ml} / \mathrm{min}$ and the running temperature was $45^{\circ} \mathrm{C}$.

\section{Additional file}

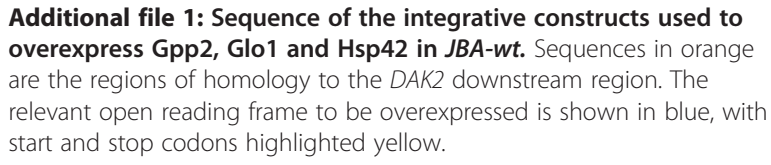

\section{Abbreviations}

ACN: Acetonitrile; CAD: Charged aerosol detector; CID: Collision induced dissociation; DAK2: Dihydroxy acetone kinase; ES: Ergosterol; FASP: Filter aided sample preparation; FTMS: Fourier transform mass spectrometry; HAP4: Heme activator protein; HPLC: High-performance liquid chromatography; HCD: High energy collision dissociation; HXK2: Hexokinase; JBA-mut: Mutated strain; JBA-wt: Wild type strain; LC: Liquid chromatography; MMTS: Methyl methanethiosulfonate; MS: Mass spectrometry;

PA: Phosphatidic acid; PC: Phosphatidylcholine:

PE: Phosphatidylethanolamine; PI: Phosphatidylinositol; PS: Phosphatidylserine; SCX: Strong cation exchange chromatography; SE: Steryl ester; TCEP: Tris(2-carboxyethyl)phosphine; TDH3: Triosephosphate dehydrogenase; TEAB: Triethylammoniumbicarbonate; TMT: Tandem mass tag; TAG: Triacylglycerol

Competing interests

The authors declare that they have no competing interests. 


\section{Authors' contributions}

PG performed all the experiments and took part in planning, writing and interpretation of results. JN and CL took part in planning, writing and interpretation of results. All authors read and approved the final manuscript.

\section{Acknowledgements}

The project was funded by Swedish Energy Agency (project no. 32918-1) and Chalmers Energy Area of Advance, which is thankfully acknowledged We also gratefully acknowledge the Proteomics Core Facility at the Sahlgrenska Academy, University of Gothenburg for performing the mass spectrometry work on the LTQ-Orbitrap XL, the purchase of which was made possible through a grant from the Knut and Alice Wallenberg Foundation (KAW2007.0118) to Gunnar C. Hansson. We would also like to thank Antonio Roldão and Luis Caspeta-Guadarrama for fruitful discussions.

Received: 28 February 2013 Accepted: 1 July 2013

Published: 15 July 2013

\section{References}

1. Dürre P. Biobutanol: An attractive biofuel. Biotechnol J 2007, 2:1525-1534.

2. Minteer S: Alcoholic fuels. 6000 Broken Sound Parkway NW, USA: CRC Press Taylor \& Francis Group; 2006

3. Savage DF, Way J, Silver PA: Defossiling fuel: how synthetic biology can transform biofuel production. ACS Chem Biol 2008, 3:13-16.

4. Zverlov W, Berezina O, Velikodvorskaya GA, Schwarz WH: Bacterial acetone and butanol production by industrial fermentation in the Soviet Union: use of hydrolyzed agricultural waste for biorefinery. Appl Microbiol Biotechnol 2006, 71:587-597.

5. Jones DT, Woods DR: Acetone-butanol fermentation revisited. Microbiol Rev 1986, 50:484-524.

6. Dürre P: New insights and novel developments in clostridial acetone/ butanol/isopropanol fermentation. Appli Microbiol Biotechnol 1998, 49:639648

7. Ezeji TC, Qureshi N, Blaschek HP: Bioproduction of butanol from biomass: from genes to bioreactors. Curr Opin Biotechnol 2007, 18:220-227.

8. Atsumi S, Cann AF, Connor MR, Shen CR, Smith KM, Brynildsen MP, Chou KJ, Hanai T, Liao JC: Metabolic engineering of Escherichia coli for 1-butanol production. Metab Eng 2008, 10:305-311.

9. Atsumi S, Hanai T, Liao JC: Non-fermentative pathways for synthesis of branched-chain higher alcohols as biofuels. Nat 2008, 451:86-89.

10. Inui M, Suda M, Kimura S, Yasuda K, Suzuki H, Toda H, Yamamoto S, Okino S, Suzuki N, Yukawa H: Expression of Clostridium acetobutylicum butanol synthetic genes in Escherichia coli. Appl Microbiol Biotechnol 2008, 77:1305-1316.

11. Steen EJ, Chan R, Prasad N, Myers S, Petzold CJ, Redding A, Ouellet M, Keasling JD: Metabolic engineering of Saccharomyces cerevisiae for the production of n-butanol. Microb Cell Fact 2008, 7:1475-2859.

12. Chen X, Nielsen KF, Borodina I, Kielland-Brandt MC, Karhumaa K: Increased isobutanol production in Saccharomyces cerevisiae by overexpression of genes in valine metabolism. Biotechnol Biofuels 2011, 4:1754-6834.

13. Steen EJ, Chan R, Prasad N, Myers S, Petzold CJ, Redding A, Ouellet M, Keasling JD: Metabolic engineering of Saccharomyces cerevisiae for the production of n-butanol. Microb Cell Fact 2008, 7:36

14. Fischer CR, Klein-Marcuschamer D, Stephanopoulos G: Selection and optimization of microbial hosts for biofuels production. Metab Eng 2008 10:295-304

15. Alsaker KV, Paredes C, Papoutsakis ET: Metabolite stress and tolerance in the production of biofuels and chemicals: Gene-expression-based systems analysis of butanol, butyrate, and acetate stresses in the anaerobe Clostridium acetobutylicum. Biotechnol Bioeng 2010, 105:11311147

16. Dunlop MJ: Engineering microbes for tolerance to next-generation biofuels. Biotechnol Biofuels 2011, 4:1754-6834

17. Ezeji TC, Qureshi N, Blaschek HP: Butanol fermentation research: upstream and downstream manipulations. Chem Rec 2004, 4:305-314

18. Gray WD, Sova C: Relation of molecule size and structure to alcohol inhibition of glucose utilization by yeast. J Bacteriol 1956, 72:349-356.

19. Butanol toxicity in the butylic fermentation: Butanol toxicity in the butylic fermentation. Biotechnol Bioeng Symp 1981, 11:567-579.

20. Bowles LK, Ellefson WL: Effects of butanol on Clostridium acetobutylicum. Appl Environ Microbiol 1985, 50:1165-1170.
21. Hui FK, Barton PG: Mesomorphic behaviour of some phospholipids with aliphatic alcohols and other non-ionic substances. Biochim Biophys Acta 1973, 296:510-517.

22. Ingram LO: Adaptation of membrane lipids to alcohols. J Bacterio/ 1976, 125:670-678.

23. Paterson SJ, Butler KW, Huang P, Labelle J, Smith IC, Schneider H: The effects of alcohols on lipid bilayers: a spin label study. Biochim Biophys Acta 1972, 266:597-602.

24. Sheetz MP, Singer SJ: Biological membranes as bilayer couples. A molecular mechanism of drug-erythrocyte interactions. Proc Natl Acad Sci USA 1974, 71:4457-4461.

25. Vollherbst-Schneck K, Sands JA, Montenecourt BS: Effect of butanol on lipid composition and fluidity of Clostridium acetobutylicum ATCC 824. Appl Environ Microbiol 1984, 47:193-194.

26. Grisham CM, Barnett RE: The effects of long-chain alcohols on membrane lipids and the (Na++K+)-ATPase. Biochim Biophys Acta 1973, 311:417-422.

27. Leao C, van Uden N: Effects of ethanol and other alkanols on the glucose transport system of Saccharomyces cerevisiae. Biotechnol Bioeng 1982, 24:2601-2604

28. Carlsen HN, Degn H, Lloyd D: Effects of alcohols on the respiration and fermentation of aerated suspensions of baker's yeast. J Gen Microbiol 1991, 137:2879-2883.

29. Iglesias R, Ferreras JM, Arias FJ, Munoz R, Girbes T: Effect of continued exposition to ethanol on activity of the ammonium and fructose transport systems in Saccharomyces cerevisiae var. ellipsoideus. Biotechnol Bioeng 1991, 37:389-391.

30. Graca Da Silveira M, Vitoria San Romao M, Loureiro-Dias MC, Rombouts FM, Abee T: Flow cytometric assessment of membrane integrity of ethanolstressed Oenococcus oeni cells. Appl Environ Microbiol 2002, 68:6087-6093

31. Ashe MP, Slaven JW, De Long SK, Ibrahimo S, Sachs AB: A novel elF2Bdependent mechanism of translational control in yeast as a response to fusel alcohols. EMBO J 2001, 20:6464-6474.

32. Albers $E_{1}$ Larsson $\mathrm{C}: \mathrm{A}$ comparison of stress tolerance in YPD and industrial lignocellulose-based medium among industrial and laboratory yeast strains. J Ind Microbiol Biotechnol 2009, 36:1085-1091.

33. Gasch AP, Spellman PT, Kao CM, Carmel-Harel O, Eisen MB, Storz G, Botstein $D$, Brown PO: Genomic expression programs in the response of yeast cells to environmental changes. Mol Biol Cell 2000, 11:4241-4257.

34. Norbeck J, Pahlman AK, Akhtar N, Blomberg A, Adler L: Purification and characterization of two isoenzymes of DL-glycerol-3-phosphatase from Saccharomyces cerevisiae. Identification of the corresponding GPP1 and GPP2 genes and evidence for osmotic regulation of Gpp2p expression by the osmosensing mitogen-activated protein kinase signal transduction pathway. J Biol Chem 1996, 271:13875-13881.

35. Stanley D, Bandara A, Fraser S, Chambers PJ, Stanley GA: The ethanol stress response and ethanol tolerance of Saccharomyces cerevisiae. J Appl Microbiol 2010, 109:13-24.

36. Inoue $Y$, Tsujimoto $Y$, Kimura A: Expression of the glyoxalase I gene of Saccharomyces cerevisiae is regulated by high osmolarity glycerol mitogen-activated protein kinase pathway in osmotic stress response. J Biol Chem 1998, 273:2977-2983.

37. Zingaro KA, Terry Papoutsakis E: GroESL overexpression imparts Escherichia coli tolerance to i-, n-, and 2-butanol, 1,2,4-butanetriol and ethanol with complex and unpredictable patterns. Metab Eng 2013, 15:196-205

38. Wagner I, Arlt H, van Dyck L, Langer T, Neupert W: Molecular chaperones cooperate with PIM1 protease in the degradation of misfolded proteins in mitochondria. EMBO J 1994, 13:5135-5145.

39. Specht S, Miller SBM, Mogk A, Bukau B: Hsp42 is required for sequestration of protein aggregates into deposition sites in Saccharomyces cerevisiae. J Cell Biol 2011, 195:617-629.

40. Kim JM, Vanguri S, Boeke JD, Gabriel A, Voytas DF: Transposable elements and genome organization: a comprehensive survey of retrotransposons revealed by the complete Saccharomyces cerevisiae genome sequence. Genome Res 1998, 8:464-478.

41. Kim TY, Ha CW, Huh WK: Differential subcellular localization of ribosomal protein L7 paralogs in Saccharomyces cerevisiae. Mol Cells 2009, 27:539-546

42. Yoshikawa K, Tanaka T, Furusawa C, Nagahisa K, Hirasawa T, Shimizu H: Comprehensive phenotypic analysis for identification of genes affecting 
growth under ethanol stress in Saccharomyces cerevisiae. FEMS Yeast Res 2009, 9:32-44.

43. Aguilera A, Benitez T: Role of mitochondria in ethanol tolerance of Saccharomyces cerevisiae. Arch Microbiol 1985, 142:389-392.

44. Gonzalez-Ramos D, van den Broek M, van Maris AJ, Pronk JT, Daran JM: Genome-scale analyses of butanol tolerance in Saccharomyces cerevisiae reveal an essential role of protein degradation. Biotechnol Biofuels 2013, 6:1754-6834.

45. Blom J, De Mattos MJT, Grivell LA: Redirection of the Respiro-Fermentative Flux Distribution in Saccharomyces cerevisiae by Overexpression of the Transcription Factor Hap4p. Appl Environ Microbiol 2000, 66:1970-1973.

46. Baer SH, Blaschek HP, Smith TL: Effect of Butanol Challenge and Temperature on Lipid Composition and Membrane Fluidity of ButanolTolerant Clostridium acetobutylicum. Appl Environ Microbiol 1987, 53:2854-2861.

47. Dickey AN, Yim WS, Faller R: Using ergosterol to mitigate the deleterious effects of ethanol on bilayer structure. J Phys Chem B 2009, 113:23882397.

48. Huffer S, Clark ME, Ning JC, Blanch HW, Clark DS: Role of alcohols in growth, lipid composition, and membrane fluidity of yeasts, bacteria, and archaea. Appl Environ Microbiol 2011, 77:6400-6408.

49. Khoomrung S, Chumnanpuen P, Jansa-Ard S, StåhIman M, Nookaew I, Boren J, Nielsen JB: Rapid Quantification of Yeast Lipid using Microwaveassisted Total Lipid Extraction and HPLC-CAD. Anal Chem 2013, 85:49124919.

50. Larsson C, Nilsson A, Blomberg A, Gustafsson L: Glycolytic flux is conditionally correlated with ATP concentration in Saccharomyces cerevisiae: a chemostat study under carbon- or nitrogen-limiting conditions. J Bacteriol 1997, 179:7243-7250.

51. Ansell R, Granath K, Hohmann S, Thevelein JM, Adler L: The two isoenzymes for yeast NAD +-dependent glycerol 3-phosphate dehydrogenase encoded by GPD1 and GPD2 have distinct roles in osmoadaptation and redox regulation. EMBO J 1997, 16:2179-2187.

52. Wisniewski JR, Zougman A, Nagaraj N, Mann M: Universal sample preparation method for proteome analysis. Nat Meth 2009, 6:359-362.

doi:10.1186/1754-6834-6-101

Cite this article as: Ghiaci et al:: Physiological adaptations of Saccharomyces cerevisiae evolved for improved butanol tolerance. Biotechnology for Biofuels 2013 6:101.

\section{Submit your next manuscript to BioMed Central and take full advantage of:}

- Convenient online submission

- Thorough peer review

- No space constraints or color figure charges

- Immediate publication on acceptance

- Inclusion in PubMed, CAS, Scopus and Google Scholar

- Research which is freely available for redistribution

Submit your manuscript at www.biomedcentral.com/submit
() BioMed Central 